Check for updates

Cite this: RSC Adv., 2019, 9, 26393

\title{
A catalyst- and solvent-free protocol for the sustainable synthesis of fused $4 \mathrm{H}$-pyran derivatives $\dagger$
}

\begin{abstract}
Md. Musawwer Khan, (iD *a Sumbulunnisan Shareef, ${ }^{a}$ Saigal $^{a}$ and Subash C. Sahoo iD b
An efficient and cost-effective method was developed for the synthesis of two kinds of fused $4 \mathrm{H}$-pyran derivatives, namely, dihydropyrano[2,3-c]pyrazole 4 and pyrano[3,2-c]chromenone 6 . The reactions of 3-methyl-1-phenyl-5-pyrazolone/4-hydroxycoumarin with aromatic aldehydes and (E)-N-methyl-1(methylthio)-2-nitroethenamine (NMSM), involving the Knoevenagel, Michael-addition, $\mathrm{O}$-cyclization and elimination reactions under thermal heating, afforded the desired products. The synthesized compounds were characterized by standard spectroscopic techniques. Further, the structures of pyrazole-fused $4 \mathrm{H}$ pyran $4 \mathrm{a}$ and coumarin-fused $4 \mathrm{H}$-pyran $6 \mathrm{~b}$ were confirmed by single-crystal XRD analysis. The short reaction time, good-to-excellent yields, elimination of the use of expensive, metallic and toxic catalysts or hazardous organic solvents and high atom-economy are some noteworthy features of this protocol.
\end{abstract}

Received 11th June 2019

Accepted 2nd August 2019

DOI: $10.1039 / c 9 r a 04370 e$

rsc.li/rsc-advances

properties. ${ }^{6}$ Among the fused heterocycles, pyrazole- and

\section{Introduction}

Recent advances in science and technology are focusing on the development of approaches that prevent pollution and minimize the usage of non-renewable resources. Recently, green techniques and processes, which obey green chemistry principles have gained significant ecological and economic interest. ${ }^{1}$ Green chemistry not only deals with processes that reduce the generation of hazardous chemicals but also introduces modern synthetic approaches, wherein synthetic chemists construct compounds more ecologically and proficiently. ${ }^{2}$

In this context, catalyst- and solvent-free multi-component syntheses have appeared as the most powerful strategy in contemporary situations, as they fulfill maximum principles of green chemistry. ${ }^{3}$ Multicomponent domino reactions (MDRs) are convergent organic reactions, in which three or more reactant molecules with multiple bond-forming tendencies and high atom-economy combine in one pot without the isolation of undesirable intermediates. ${ }^{4}$ Therefore, MDR strategies have been explored to access functionalized organic compounds under green-chemistry conditions and are preferred over stepwise synthetic strategies. ${ }^{5}$

Fused heterocycles represent a dynamic class of organic compounds with notable medicinal and agrochemical

\footnotetext{
${ }^{a}$ Department of Chemistry, Aligarh Muslim University, Aligarh, 202002, India. E-mail: musawwer@gmail.com

${ }^{b}$ Department of Chemistry, Center of Advanced Studies in Chemistry, Panjab University, Chandigarh-160014, India

$\dagger$ Electronic supplementary information (ESI) available. CCDC 1901104 and 1901105. For ESI and crystallographic data in CIF or other electronic format see DOI: $10.1039 / \mathrm{c} 9 \mathrm{ra04370e}$
} coumarin-fused oxygen-containing heterocyclic systems, particularly $4 H$-pyran derivatives, are omnipresent in several natural and unnatural bioactive molecules with promising properties both in pharmacological and materials sciences. ${ }^{7}$ Pyrazole-fused $4 H$-pyran derivatives have shown several biological activities such as antimalarial, ${ }^{8}$ antibacterial, ${ }^{9}$ antimicrobial, ${ }^{10}$ anti-inflammatory, ${ }^{11}$ anti-tubercular, ${ }^{12}$ hypoglycemic and vasodilator activities..$^{13}$ On the other hand, coumarin-fused $4 H$-pyrans also exhibit several activities like antioxidant, ${ }^{\mathbf{1 4}}$ antifungal ${ }^{15}$ and anticancer. ${ }^{16}$ Fig. 1 provides a glimpse of fused $4 H$-pyran-based biologically and medicinally active compounds. For example, compounds I and II have antibacterial characteristics, ${ }^{17}$ whereas, compounds $\mathbf{I I I}$ and IV possess antifungal activity. ${ }^{18}$

Recently, (E)- $N$-methyl-1-(methylthio)-2-nitroethenamine (NMSM) was explored as a fascinating building block for the construction of diverse heterocyclic compounds. ${ }^{19}$ The starting material NMSM has a polarizable ethylene skeleton, bearing electrophilic and nucleophilic centers at the two ends, as well as electron-withdrawing $\left(-\mathrm{NO}_{2}\right)$, electron-donating $\left(\mathrm{CH}_{3} \mathrm{NH}-\right)$ and good leaving groups (methylsulfanyl), where each of them plays an important role in the formation of products.

A literature scan revealed that the synthesis of pyrazole-fused $4 \mathrm{H}$-pyran was performed previously using $\mathrm{InCl}_{3}$ (ref. 20) and piperidine, ${ }^{21}$ whereas coumarin-fused $4 H$-pyran scaffolds were prepared using catalysts such as STA, ${ }^{22} 6,6^{\prime}$-thiobis(methylene)$\beta$-cyclodextrin dimer ${ }^{23}$ and piperidine. ${ }^{24}$ Regardless of the adequacy of these reported methods, each exhibits one or more shortcomings, such as the required usage of catalysts ${ }^{\mathbf{2 0}-24}$ or solvents ${ }^{20,24}$ and prolonged reaction time. ${ }^{20,24}$ Therefore, there still remains a scope of improvement of the synthetic strategy to 


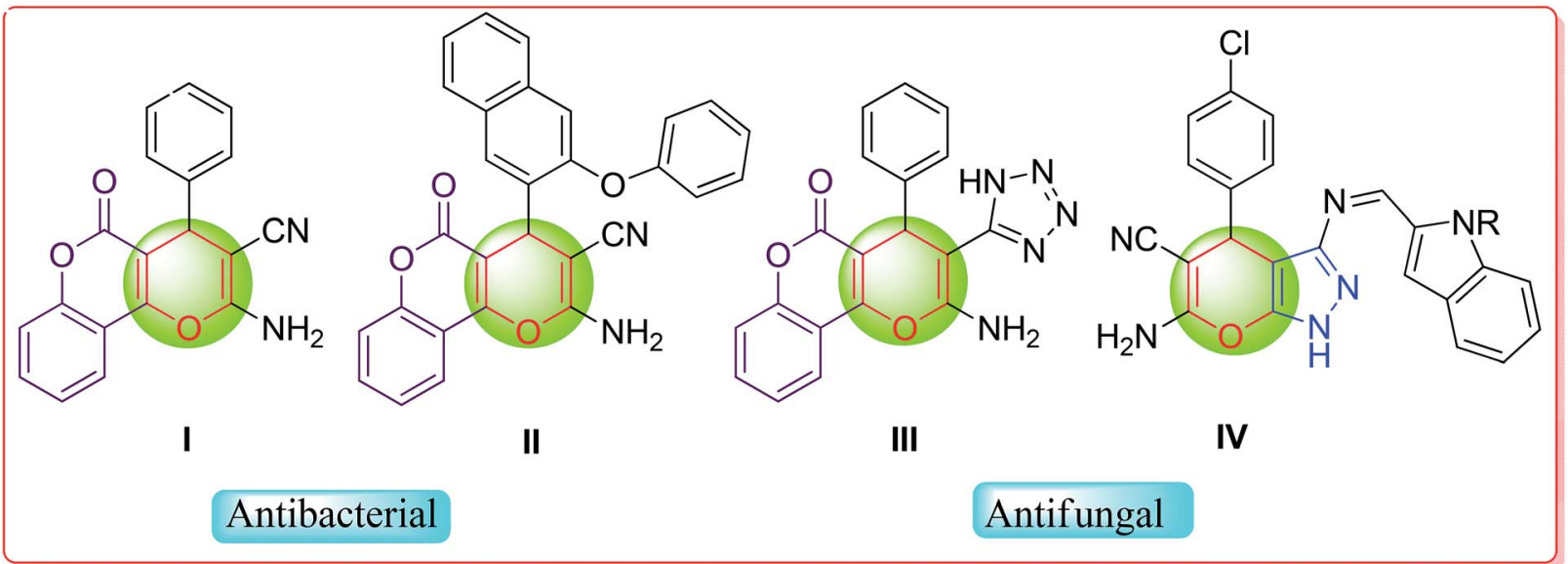

Fig. 1 Some representative examples of biologically active fused $4 H$-pyrans.

access such important fused $4 H$-pyrans. In continuation of our effort to develop green and sustainable methodologies for the synthesis of heterocycles by using NMSM and other building blocks ${ }^{25,26}$ we herein intended to utilize the distinctive reactivity of NMSM and aromatic aldehydes with 3-methyl-1-phenyl-5pyrazolone/4-hydroxycoumarin for the synthesis of fused $4 H^{-}$ pyran, as depicted in Scheme 1.

\section{Results and discussion}

For the present study, a model reaction was initially conducted between NMSM $1(1.0 \mathrm{mmol})$, benzaldehyde $2(1.0 \mathrm{mmol})$ and 3methyl-1-phenyl-5-pyrazolone $3(1.0 \mathrm{mmol})$ in the presence of $10 \mathrm{~mol} \%$ of $\mathrm{ZnO}$ nano-catalyst under refluxing ethanol $(4 \mathrm{~mL})$ (Table 1, entry 1 ). The progress of the reaction was monitored by thin layer chromatography (TLC). After the completion of the reaction, the product $\mathbf{4 a}$ was isolated in $60 \%$ yield by simple filtration. The structure of the compound $4 \mathbf{a}$ was confirmed by its melting point, IR, ${ }^{1} \mathrm{H}$ and ${ }^{13} \mathrm{C}$ NMR spectroscopies, and the data matched well with the reported data. ${ }^{20-24}$ Encouraged by these results, we proceeded to optimize the reaction conditions. For this process, the model reaction was executed in the presence of different acid and base catalysts $(10 \mathrm{~mol} \%)$ in EtOH (4 $\mathrm{mL}$ ) as well as without catalyst in EtOH with reflux (Table 1, entries 2-6). Unfortunately, we did not observe any improvement in the results. Therefore, the original reaction was performed without any catalyst or solvent at $80^{\circ} \mathrm{C}$ (Table 1, entry 7).
Amusingly, the reaction afforded better results in terms of reaction time and yield.

In addition, the above reaction was executed at different temperatures ranging from 90 to $130{ }^{\circ} \mathrm{C}$ under catalyst- and solvent-free conditions (Table 1, entries $8-12$ ) to assess the effect of temperature. We observed that maximum yields were obtained at $110{ }^{\circ} \mathrm{C}$ within a short time period, and no further increase in the yield occurred with an increase in the temperature. Thus, the solvent-free and catalyst-free reaction at $110^{\circ} \mathrm{C}$ emerged as the optimized condition for the present protocol for the synthesis of compound $\mathbf{4 a}$.

Similar optimal conditions were found for the synthesis of compound 6a by performing a series of reactions between 4hydroxycoumarine, benzaldehyde and NMSM, which are also summarized in Table 1.

After determining the optimized conditions, we focused on establishing the substrate scope. A reaction was performed between compound 3, 4-chlorobenzaldehyde and NMSM under the optimized reaction conditions, which gave the desired product $\mathbf{4 b}$ with $85 \%$ yield (Table 2 ).

Next, using the optimized reaction conditions, reactions were performed with other aromatic aldehydes having substituents such as 4-Br, 4-F, 4-Me, 4-Et, 4- $\mathrm{NO}_{2}$, 4-OMe, 3-Cl, 2-Cl, 3$\mathrm{Br}, 3-\mathrm{NO}_{2}, 3,4-(\mathrm{OMe})_{2}$, and 3,4,5-(OMe $)_{3}$ with 3-methyl-1-phenyl5-pyrazolone and NMSM. The reactions afforded corresponding products $4 \mathbf{c}-\mathbf{4 n}$ with good-to-excellent yields. Similarly, this reaction was also performed with a hetero-aromatic aldehyde,<smiles>[R]c1ccc(C2C([N+](=O)[O-])=C(NC)Oc3c2c(=O)oc2ccccc32)cc1</smiles>

Scheme 1 Synthesis of pyrazole- and coumarin-fused 4H-pyrans under neat conditions. 
Table 1 Optimization of reaction conditions for the synthesis of compounds $4 a$ and $6 a^{a}$

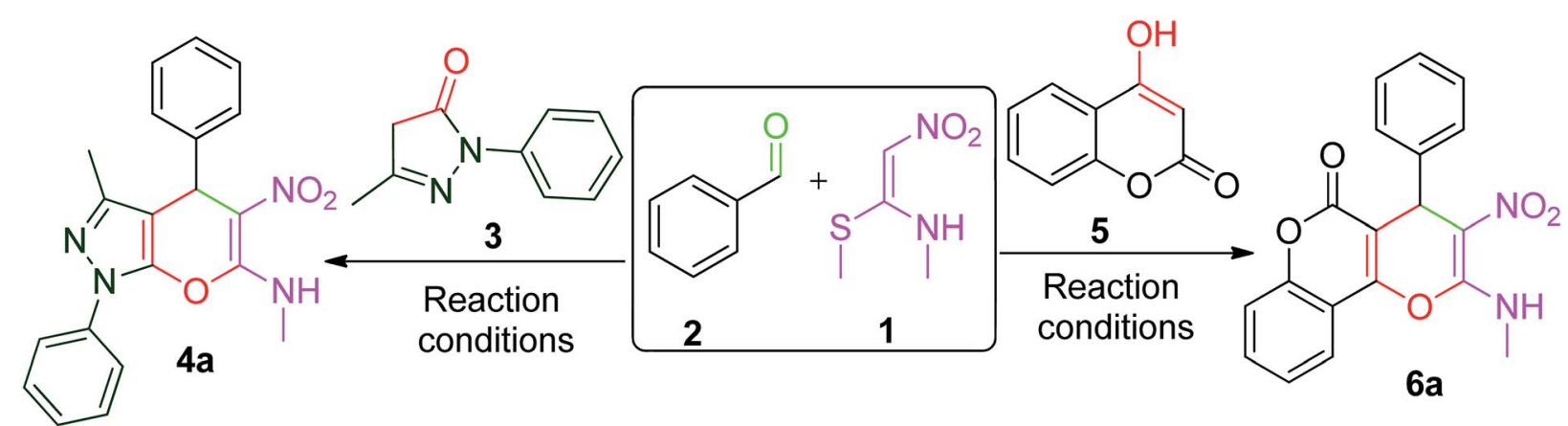

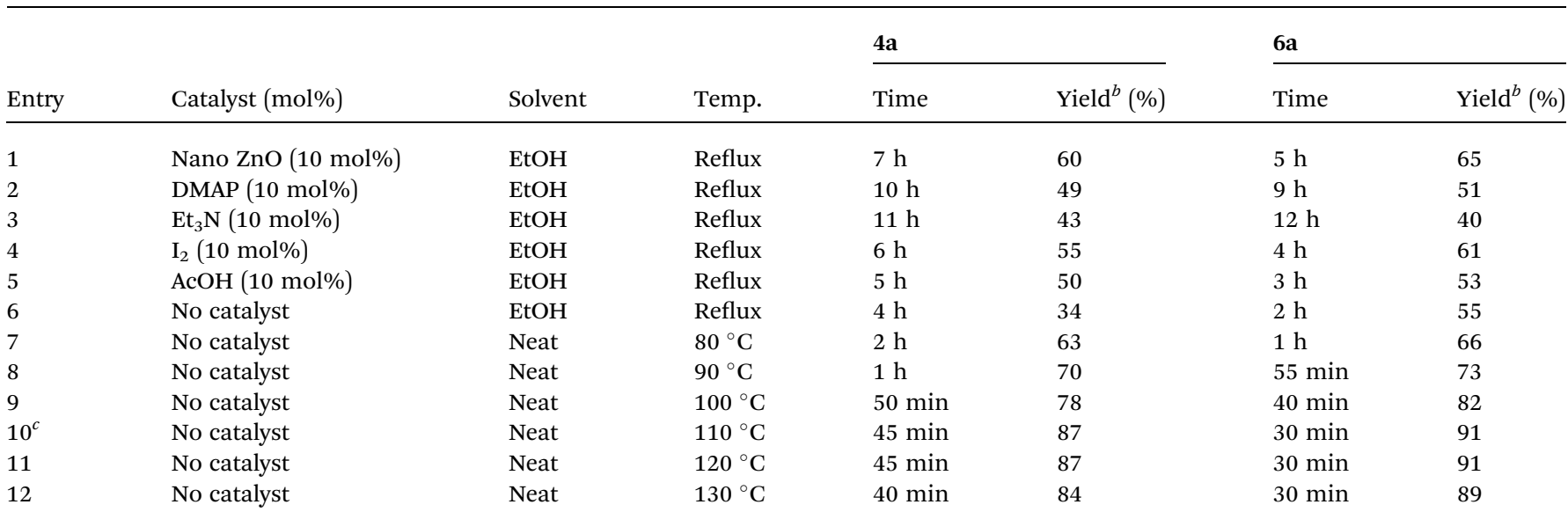

${ }^{a}$ Reaction conditions: 3-methyl-1-phenyl-5-pyrazolone/4-hydroxy coumarin $(1 \mathrm{mmol})$, benzaldehyde $(1 \mathrm{mmol})$ and NMSM $(1 \mathrm{mmol}) .{ }^{b}$ Isolated yields. ${ }^{c}$ Optimized reaction conditions.

i.e. pyridine-3-carbaldehyde, which afforded the expected product 40 in $83 \%$ yield.

Further, to investigate the generality and applicability of the above protocol, we executed the reaction by replacing reactant 3 with 4-hydroxycoumarin and $\mathbf{5}$ using aromatic aldehydes tethered to substituents like 4-Cl, 4-Br, 4-F, 4-Me, 4-OMe, 4- $\mathrm{NO}_{2}$, 4Et, 3,4-diOMe, 3,4,5-triOMe, 3- $\mathrm{NO}_{2}, 3-\mathrm{Cl}, 2-\mathrm{NO}_{2}$ and NMSM under similar optimized conditions, which afforded the desired coumarin-fused $4 H$-pyrans $\mathbf{6 b}-\mathbf{m}$ in good yields, as shown in Table 3. Aldehydes possessing both electron-withdrawing groups as well as electron-donating groups afforded products smoothly. The reaction of 4-hydroxycoumarin was also performed with thiophene-2-carbaldehyde and NMSM, which furnished the product $\mathbf{6 n}$ in good yield.

All the synthesized compounds were characterized by spectroscopic techniques including IR, ${ }^{1} \mathrm{H}$ and ${ }^{13} \mathrm{C}$ NMR spectroscopies and elemental analysis; the resulting data for several of the reported compounds are well-matched with the literature. ${ }^{\mathbf{2 0 - 2 4}}$ Further, the structure and stereochemistry of the compounds 4a (CCDC 1901104) and 6b (CCDC 1901105) were unambiguously confirmed by single-crystal X-ray diffraction analysis, and the ORTEP diagrams are shown in Fig. 2 and 3, respectively.

Single crystals of the compounds $\mathbf{4 a}$ and $\mathbf{6 b}$ were mounted on a glass fiber and utilized for XRD data collection. Light yellow and white-colored crystals of compounds $\mathbf{4 a}$ and $\mathbf{6 b}$, respectively, were grown by the slow evaporation of $\mathrm{CH}_{3} \mathrm{CN}$ solvent for X-ray analysis. The data were obtained from a Super Nova, single source at offset/far and HyPix3000 diffractometer using graphite-monochromated $\mathrm{Mo}(\mathrm{K} \alpha)$ radiation $(\lambda=0.71073$ A) at $293 \mathrm{~K}$.

The crystallized compound of $\mathbf{4 a}$ has a monoclinic crystal system, with the space group $P 2_{1} / c$ and the unit cell dimensions $a=10.7875(3) \AA, b=20.8262(4) \AA, c=8.0335(2) \AA$. On the other hand, the crystallized compound of $\mathbf{6 b}$ also has a monoclinic crystal system with the space group $P 2_{1} / c$ and unit cell dimensions $a=8.5412(2) \AA, b=13.1791(3) \AA, c=14.8906(3) \AA$.

Finally, the formation of the products can be rationalized via a domino reaction involving Knoevenagel condensation, Michael-addition, $\mathrm{O}$-cyclization and elimination mechanisms, as illustrated in Scheme 2 .

The first step is a simple Knoevenagel condensation of an aromatic aldehyde with a 1,3-dinuleophilic oxygen-containing compound (4-hydroxycoumarin or 3-methyl-1-phenyl-5pyrazolone) to generate an intermediate I. The intermediate I undergoes a Michael-addition reaction with NMSM to form species II. Then, species II affords the desired product $\mathbf{4} / \mathbf{6}$ by an intra-molecular $O$-cyclization followed by the elimination of -MeSH via species III. 
<smiles></smiles>

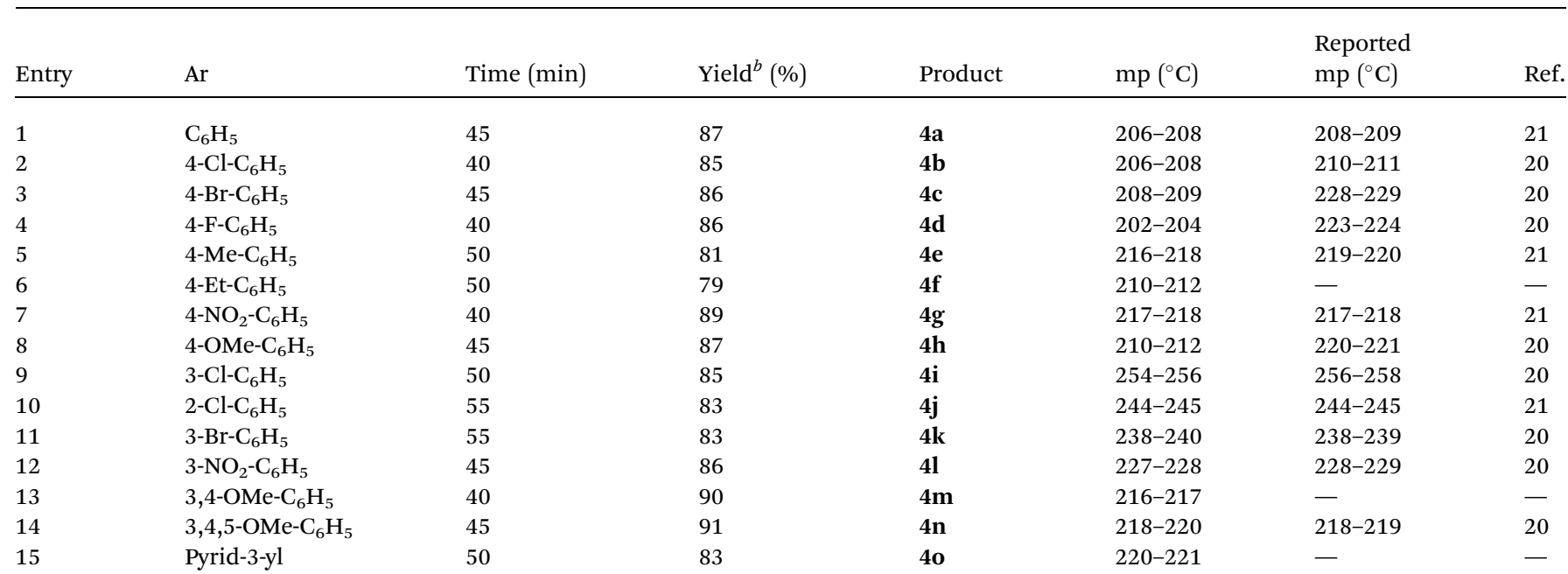

${ }^{a}$ Reaction conditions: 3-methyl-1-phenyl-5-pyrazolone $(1 \mathrm{mmol})$, aryl aldehydes $(1 \mathrm{mmol})$, NMSM $(1 \mathrm{mmol})$ under neat conditions at $110{ }^{\circ} \mathrm{C}$. ${ }^{b}$ Isolated yields.

\section{Conclusion}

In summary, we have successfully developed a practical and green method for the synthesis of highly functionalized pyrazole- and coumarin-fused $4 H$-pyrans by the reaction of NMSM or aryl aldehydes with 3-methyl-1-phenyl-5-pyrazolone or 4hydroxycoumarin, respectively, under thermal heating and neat conditions. The presented protocol is characterized by important features such as a short reaction time, good-to-excellent yields, elimination of the use of toxic catalysts or solvents, easy isolation of compounds without traditional column purification and applicability to a broad range of substrates.

\section{Experimental}

\section{General}

4-Hydroxycoumarin, 3-methyl-1-phenyl-5-pyrazolone, NMSM and all the aldehydes were procured from Sigma-Aldrich. All the solvents were obtained from Merck and Otto Chemie. All the reactions were completed in a REMI $2 \mathrm{MLH}$ thermo-mechanical stirrer. TLC analysis was carried out using silica gel GF-254 from SRL (Alfa Aesar). Melting points were obtained on a Stuart digital melting point apparatus (SMP10) and are uncorrected. IR spectra were performed with potassium bromide $(\mathrm{KBr})$ pellets on a PerkinElmer 10.4.00 IR spectrophotometer. ${ }^{1} \mathrm{H}$ and ${ }^{13} \mathrm{C}$ NMR spectral analyses were performed on Bruker (Avance-II $400 \mathrm{MHz}$ ),
Varian-AS400 NMR, and Bruker Bio Spin GmbH spectrometers using tetramethylsilane (TMS) as the internal standard and DMSO- $d_{6}$ or $\mathrm{CDCl}_{3}$ as the solvent. Crystal data were collected with a Super Nova, single source at offset/far and HyPix3000 diffractometer (CCD) using graphite monochromated MoK $\alpha$ radiation $(\lambda=0.71073 \AA)$ at $296 \mathrm{~K}$. HRMS spectra were recorded on a highresolution mass spectrometer XEVO G2-XS QTOF.

\section{Typical procedure for the preparation of functionalized fused $4 H$-pyrans (4 and 6)}

A dried $5 \mathrm{~mL}$ round-bottom flask was equipped with a Tefloncoated magnet and charged with a combination of 3-methyl-1phenyl-1 $H$-pyrazol-5(4H)-one/4-hydroxycoumarin (1 $\mathrm{mmol})$, aromatic aldehyde $(1 \mathrm{mmol})$, and NMSM $(1 \mathrm{mmol})$. The mixture of all the reagents was heated at $110{ }^{\circ} \mathrm{C}$ with stirring for a specified time under neat conditions. The progress of the reactions was monitored by TLC. After completion of the reaction as indicated by TLC, the resulting precipitate was cooled, and $2 \mathrm{~mL}$ of ethanol was added and stirred for $5 \mathrm{~min}$. Next, the precipitate was filtered and washed with $3 \mathrm{~mL}$ cold ethanol. Purification of the crude product was done by recrystallization from hot acetonitrile to yield the pure products.

\section{Characterization data of selected compounds}

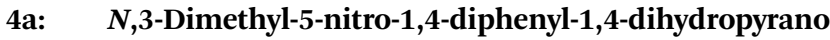
[2,3-c]pyrazol-6-amine. Isolated as yellow solid; yield: $87 \%$; $\mathrm{mp}$ 
<smiles>[R]c1ccc(C=O)cc1</smiles><smiles>CNC1=C([N+](=O)[O-])C(c2ccccc2)c2c(c3ccccc3oc2=O)O1</smiles>

$6 a, 30 \mathrm{~min}, 91 \%$<smiles>CNC1=C([N+](=O)[O-])C(c2ccc(OC)cc2)c2c(c3ccccc3oc2=O)O1</smiles>

6f, $20 \mathrm{~min}, 81 \%$<smiles>CNC1=C([N+](=O)[O-])C(c2ccc(Cl)cc2)c2c(c3ccccc3oc2=O)O1</smiles>

6b, $30 \mathrm{~min}, 92 \%$<smiles>CNC1=C([N+](=O)[O-])C(c2ccc(Br)cc2)c2c(c3ccccc3oc2=O)O1</smiles>

6c, $35 \mathrm{~min}, 87 \%$<smiles>CNC1=C([N+](=O)[O-])C(c2ccc(F)cc2)c2c(c3ccccc3oc2=O)O1</smiles>

6 d, $30 \mathrm{~min}, 86 \%$<smiles>CNC1=C([N+](=O)[O-])C(c2ccc(C)cc2)c2c(c3ccccc3oc2=O)O1</smiles>

$6 e, 35 \mathrm{~min}, 81 \%$<smiles>CNC1=C([N+](=O)[O-])C(c2ccc([N+](=O)[O-])cc2)c2c(c3ccccc3oc2=O)O1</smiles>

$6 g, 25 \min , 92 \%$<smiles>CCc1ccc(C2C([N+](=O)[O-])=C(NC)Oc3c2c(=O)oc2ccccc32)cc1</smiles>

$6 h, 40 \mathrm{~min}, 80 \%$<smiles>CNC1=C([N+](=O)[O-])C(c2ccc(OC)c(OC)c2)c2c(c3ccccc3oc2=O)O1</smiles>

6i, $25 \mathrm{~min}, 87 \%$<smiles>CNC1=C([N+](=O)[O-])C(c2cc(OC)c(OC)c(OC)c2)c2c(c3ccccc3oc2=O)O1</smiles>

6j, 40 min, 92\%<smiles>CNC1=C([N+](=O)[O-])C(c2cccc([N+](=O)[O-])c2)c2c(c3ccccc3oc2=O)O1</smiles>

6k, 40 min, $81 \%$<smiles>CNC1=C([N+](=O)[O-])C(c2cccc(Cl)c2)c2c(c3ccccc3oc2=O)O1</smiles>

$6 I, 40 \mathrm{~min}, 85 \%$<smiles>CNC1=C([N+](=O)[O-])C(c2ccccc2[N+](=O)[O-])c2c(c3ccccc3oc2=O)O1</smiles>

$6 \mathrm{~m}, 35 \mathrm{~min}, 83 \%$<smiles>CNC1=C([N+](=O)[O-])C(c2cccs2)c2c(c3ccccc3oc2=O)O1</smiles>

$6 n, 40 \mathrm{~min}, 81 \%$

\footnotetext{
${ }^{a}$ Reaction conditions: 3-methyl-1-phenyl-5-pyrazolone (1 mmol), aromatic aldehydes ( $\left.1 \mathrm{mmol}\right)$, NMSM $(1 \mathrm{mmol})$ under neat conditions at $110{ }^{\circ} \mathrm{C}$. ${ }^{b}$ Isolated yields.
}

206-208 ${ }^{\circ} \mathrm{C}$; IR (KBr, $\left.\mathrm{cm}^{-1}\right): 3173,3067,3026,2943,2920,1868$, 1655, 1519, 1449, 1390, 1265, 1126, 969, 864, 753; ${ }^{1} \mathrm{H}$ NMR (500 $\left.\mathrm{MHz}, \mathrm{CDCl}_{3}\right) \delta 2.00\left(\mathrm{~s}, 3 \mathrm{H}, \mathrm{CH}_{3}\right), 3.22\left(\mathrm{~d}, J=4.0 \mathrm{~Hz}, 3 \mathrm{H}, \mathrm{NCH}_{3}\right)$, $5.25(\mathrm{~s}, 1 \mathrm{H}, \mathrm{CH}), 7.20$ (d, J = 5.5 Hz, 1H, ArH), 7.28-7.35 (m, 5H,
ArH), 7.49 (t, $J=7.0 \mathrm{~Hz}, 2 \mathrm{H}, \operatorname{ArH}), 7.69$ (d, $J=7.5 \mathrm{~Hz}, 2 \mathrm{H}, \operatorname{ArH})$, 10.55 (s, $1 \mathrm{H}, \mathrm{NH}) ;{ }^{13} \mathrm{C} \mathrm{NMR}\left(125 \mathrm{MHz}, \mathrm{CDCl}_{3}\right) \delta 12.7,28.4,38.4$, $101.1,110.1,120.7,126.8,126.9,128.0,128.2$, 129.3, 137.4, 141.9, 146.4, 159.2; anal. calcd for $\mathrm{C}_{20} \mathrm{H}_{18} \mathrm{~N}_{4} \mathrm{O}_{3}$ : C, 66.29; H, 


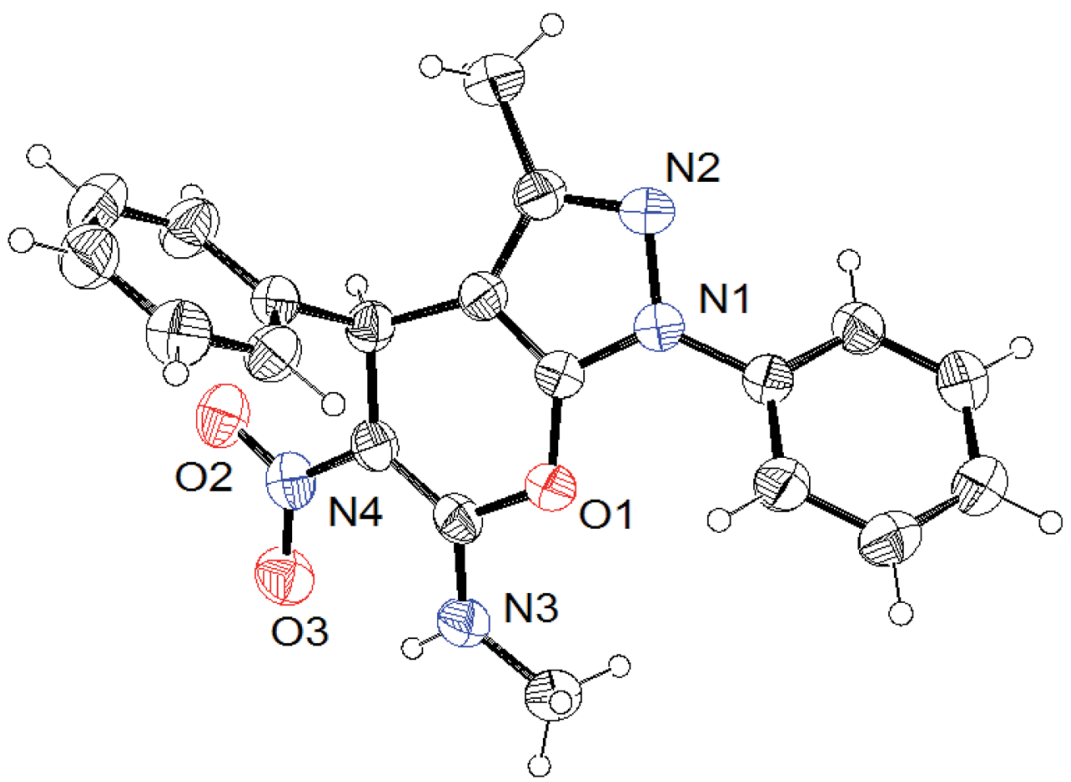

Fig. 2 ORTEP representation of compound 4a (CCDC 1901104).

5.01; N, 15.46\%. Found: C, 66.19; H, 5.05; N, 15.39\%. EI-HRMS: anal. calcd for $\left[\mathrm{C}_{20} \mathrm{H}_{18} \mathrm{~N}_{4} \mathrm{O}_{3}+\mathrm{H}^{+}\right]$: anal. calcd: 363.1412 , found: 363.1452 .

4b: 4-(4-Chlorophenyl)- $N, 3-d i m e t h y l-5-n i t r o-1-p h e n y l-1,4-$ dihydropyrano[2,3-c]pyrazol-6-amine. Isolated as white solid; yield: $85 \%$; mp $206-208{ }^{\circ} \mathrm{C}$; IR $\left(\mathrm{KBr}, \mathrm{cm}^{-1}\right)$ : 3411, 3184, 3073, 2946, 2887, 1656, 1617, 1514, 1430, 1296, 1266, 1158, 1016, ${ }^{1} \mathrm{H}$ $\mathrm{NMR}\left(400 \mathrm{MHz}, \mathrm{CDCl}_{3}\right) \delta 1.98\left(\mathrm{~s}, 3 \mathrm{H}, \mathrm{CH}_{3}\right), 3.23(\mathrm{~d}, J=5.16 \mathrm{~Hz}$, $\left.3 \mathrm{H}, \mathrm{NCH}_{3}\right), 5.23(\mathrm{~s}, 1 \mathrm{H}, \mathrm{CH}), 6.95(\mathrm{t}, J=8.0 \mathrm{~Hz}, 2 \mathrm{H}, \mathrm{ArH}), 7.25-$ $7.27(\mathrm{~m}, 2 \mathrm{H}, \mathrm{ArH}), 7.34(\mathrm{t}, J=8 \mathrm{~Hz}, 1 \mathrm{H}, \operatorname{ArH}), 7.49(\mathrm{t}, J=7.2 \mathrm{~Hz}$, 2H, ArH), 7.68 (dd, $J=8.8 \mathrm{~Hz}, 1.0 \mathrm{~Hz}, 2 \mathrm{H}, \operatorname{ArH}), 10.56$ (d, $J=$

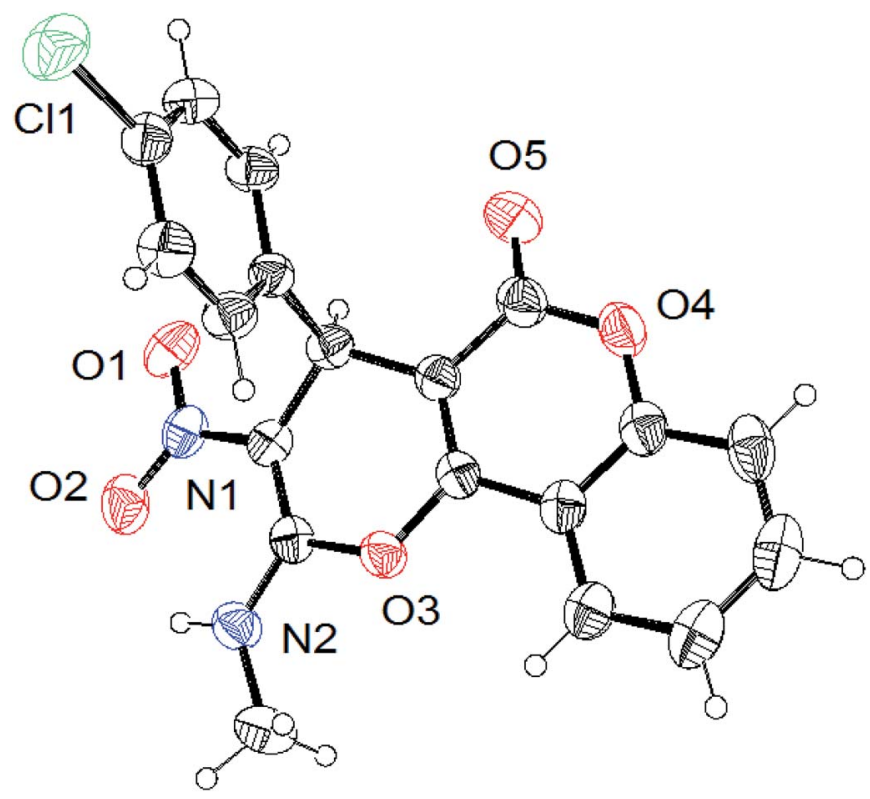

Fig. 3 ORTEP representation of compound 6b (CCDC 1901105).
$4.4 \mathrm{~Hz}, 1 \mathrm{H}, \mathrm{NH}) ;{ }^{13} \mathrm{C} \mathrm{NMR}\left(100 \mathrm{MHz}, \mathrm{CDCl}_{3}\right) \delta 12.7,28.5,37.6$, $100.8,110.3,115.0,115.2,120.7,126.9,129.4,129.8,137.3$, $141.9,146.3,154.5,159.0,160.5,163.6$; anal. calcd for $\mathrm{C}_{20} \mathrm{H}_{17} \mathrm{ClN}_{4} \mathrm{O}_{3}$ : C, 60.53; H, 4.32; N, 14.12\%. Found: C, 60.50; H, 4.36; N, $14.10 \%$.

4c: 4-(4-Bromophenyl)- $N, 3-d i m e t h y l-5-n i t r o-1-p h e n y l-1,4-$ dihydropyrano[2,3-c]pyrazol-6-amine. Isolated as white solid; yield: $86 \%$; mp 208-209 ${ }^{\circ} \mathrm{C}$; IR $\left(\mathrm{KBr}, \mathrm{cm}^{-1}\right)$ : 3433, 3185, 3068, 2923, 2368, 1654, 1518, 1388, 1232, 1125, 1012, 904; ${ }^{1} \mathrm{H}$ NMR $\left(400 \mathrm{MHz}, \mathrm{DMSO}-d_{6}\right) \delta 1.98\left(\mathrm{~s}, 3 \mathrm{H}, \mathrm{CH}_{3}\right), 3.23(\mathrm{~d}, J=5.0 \mathrm{~Hz}, 3 \mathrm{H}$, $\left.\mathrm{NCH}_{3}\right), 5.20(\mathrm{~s}, 1 \mathrm{H}, \mathrm{CH}), 7.16-7.20(\mathrm{~m}, 2 \mathrm{H}, \mathrm{ArH}), 7.34(\mathrm{~m}, 1 \mathrm{H}$, $\operatorname{ArH}), 7.40$ (d, $J=8.0 \mathrm{~Hz}, 2 \mathrm{H}, \operatorname{ArH}), 7.49$ (t, $J=7.9 \mathrm{~Hz}, 2 \mathrm{H}, \operatorname{ArH})$, 7.67 (dd, $J=8.6,1.1 \mathrm{~Hz}, 2 \mathrm{H}, \operatorname{ArH}), 10.55$ (d, $J=5.5 \mathrm{~Hz}, 1 \mathrm{H}, \mathrm{NH})$; ${ }^{13} \mathrm{C}$ NMR (100 MHz, DMSO- $\left.d_{6}\right) \delta 12.7,28.5,37.9,100.4,110.0$, $120.7,120.8,126.9$, 129.4, 129.9, 131.3, 137.3, 140.9, 141.8, 146.3, 159.0; anal. calcd for $\mathrm{C}_{20} \mathrm{H}_{17} \mathrm{BrN}_{4} \mathrm{O}_{3}$ : C, 54.44; H, 3.88; N, 12.70. Found: C, 54.34; H, 3.85; N, $12.73 \%$.

4d: 4-(4-Fluorophenyl)- $N, 3-d i m e t h y l-5-n i t r o-1-p h e n y l-1,4-$ dihydropyrano[2,3-c]pyrazol-6-amine. Isolated as white solid; yield: 86\%; mp: $202-204{ }^{\circ} \mathrm{C}$; IR $\left(\mathrm{KBr}, \mathrm{cm}^{-1}\right)$ : 3435, 2924, 2855 , 1656.7, 1513.4, 1466.6, 1364, 1231, 1048, 753, 664, 530; ${ }^{1} \mathrm{H}$ NMR $\left(400 \mathrm{MHz}, \mathrm{CDCl}_{3}\right) \delta 1.98\left(\mathrm{~s}, 3 \mathrm{H}, \mathrm{CH}_{3}\right), 3.23(\mathrm{~d}, J=5.2 \mathrm{~Hz}, 3 \mathrm{H}$, $\left.\mathrm{NCH}_{3}\right), 5.22(\mathrm{~s}, 1 \mathrm{H}, \mathrm{CH}), 7.24(\mathrm{~s}, 4 \mathrm{H}, \mathrm{ArH}), 7.34(\mathrm{t}, J=7.52 \mathrm{~Hz}$, $1 \mathrm{H}, \operatorname{ArH}), 7.49$ (t, $J=7.6 \mathrm{~Hz}, 2 \mathrm{H}, \operatorname{ArH}), 7.67(\mathrm{~d}, J=8.8 \mathrm{~Hz}, 2 \mathrm{H}$, $\mathrm{ArH}), 10.56$ (d, $J=4.8 \mathrm{~Hz}, 1 \mathrm{H}, \mathrm{NH}) ;{ }^{13} \mathrm{C} \mathrm{NMR}\left(100 \mathrm{MHz}, \mathrm{CDCl}_{3}\right.$ ) $\delta 12.8,28.5,37.8,100.5,110.1,120.8,127.0,128.4,129.4,129.5$, $132.7,137.3,140.4,141.9,146.3,159.0$; anal. calcd for $\mathrm{C}_{20} \mathrm{H}_{17} \mathrm{FN}_{4} \mathrm{O}_{3}$ : C, 63.15; H, 4.50; N, 14.73\%. Found: C, 63.10; H, 4.47; N, $14.75 \%$.

4f: 4-(4-Ethylphenyl)- $N, 3-d i m e t h y l-5-n i t r o-1-p h e n y l-1,4-d i h y-$ dropyrano[2,3-c]pyrazol-6-amine. Isolated as white solid; yield: 79\%; mp 210-212 ${ }^{\circ} \mathrm{C}$; IR ( $\left.\mathrm{KBr}, \mathrm{cm}^{-1}\right): 3435,3177,3048,3021$, 2962, 2929, 2875, 1654, 1519, 1474, 1392, 1267, 1195, 1050, 963; ${ }^{1} \mathrm{H}$ NMR $\left(400 \mathrm{MHz}, \mathrm{CDCl}_{3}\right) \delta 1.19\left(\mathrm{t}, J=7.6 \mathrm{~Hz}, 3 \mathrm{H}, \mathrm{CH}_{3}\right), 2.0(\mathrm{~s}$, 


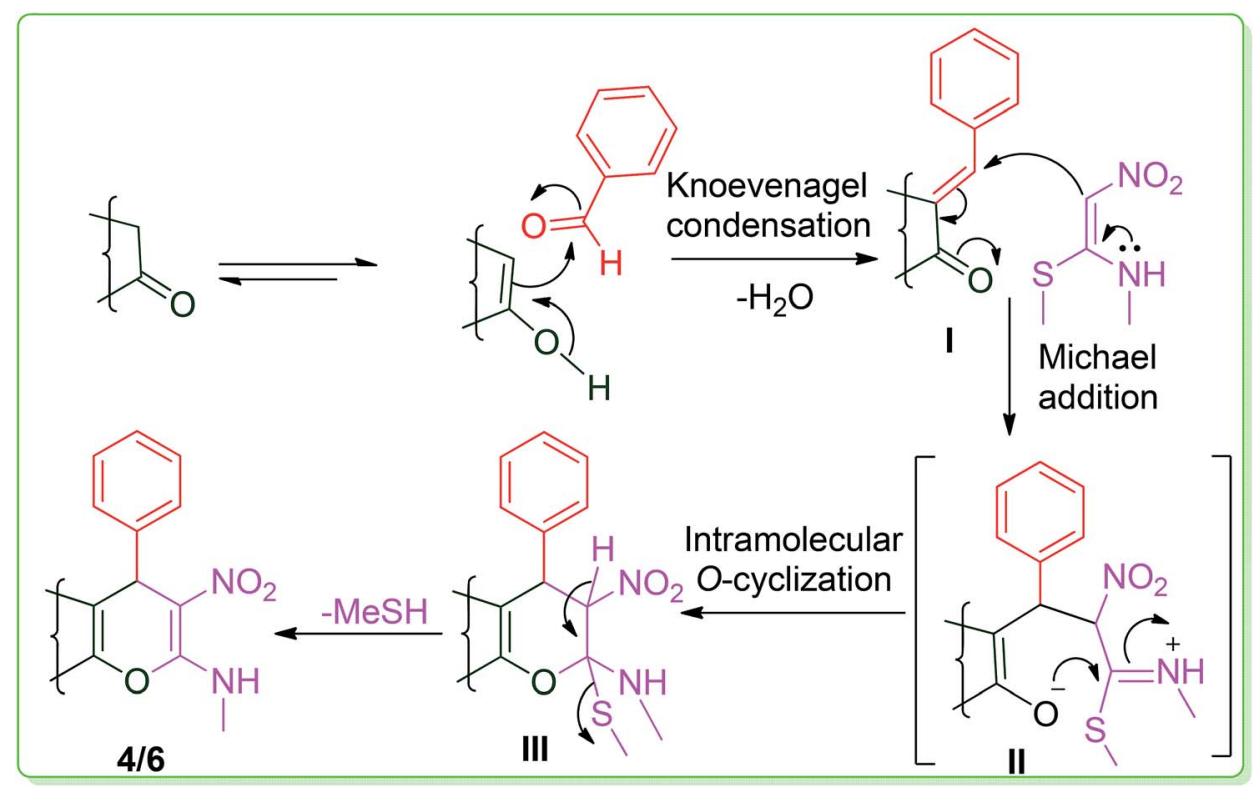

Scheme 2 Plausible mechanism for the synthesis of the pyrazole- and coumarin-fused $4 \mathrm{H}$-pyrans.

$\left.3 \mathrm{H}, \mathrm{CH}_{3}\right), 2.59\left(\mathrm{q}, J=7.6 \mathrm{~Hz}, 2 \mathrm{H}, \mathrm{CH}_{2}\right), 3.19(\mathrm{~d}, J=5.2 \mathrm{~Hz}, 3 \mathrm{H}$, $\left.\mathrm{NCH}_{3}\right), 5.22(\mathrm{~s}, 1 \mathrm{H}, \mathrm{CH}), 7.10(\mathrm{~d}, J=8.0 \mathrm{~Hz}, 2 \mathrm{H}, \mathrm{ArH}), 7.20(\mathrm{~d}, J=$ $8.0 \mathrm{~Hz}, 2 \mathrm{H}, \mathrm{ArH}), 7.30-7.34(\mathrm{~m}, 1 \mathrm{H}, \mathrm{ArH}), 7.46-7.50(\mathrm{~m}, 2 \mathrm{H}$, ArH), 7.61-7.68 (m, 2H, ArH), 10.55 (d, $J=5.2 \mathrm{~Hz}, 1 \mathrm{H}, \mathrm{NH}) ;{ }^{13} \mathrm{C}$ NMR (100 MHz, $\left.\mathrm{CDCl}_{3}\right) \delta 12.8,15.4,28.4,28.4,37.9,101.2$, $110.6,120.6,126.7,127.7,127.9$, 129.4, 137.4, 139.0, 141.8, 142.8, 146.4, 159.1; anal. calcd for $\mathrm{C}_{22} \mathrm{H}_{22} \mathrm{~N}_{4} \mathrm{O}_{3}$ : C, 67.68; $\mathrm{H}$, 5.68 ; N, 14.35\%. Found: C, 67.60; H, 5.65; N, 14.40\%.

4g: $\quad N, 3-D i m e t h y l-5-n i t r o-4-(4-n i t r o p h e n y l)-1-p h e n y l-1,4-$ dihydropyrano[2,3-c]pyrazol-6-amine. Isolated as white solid; yield: $89 \%$; mp: $217-218{ }^{\circ} \mathrm{C}$; IR $\left(\mathrm{KBr}, \mathrm{cm}^{-1}\right)$ : 3439, 2927, 1653, 1458, 1558, 1391, 1350, 1235, 1270, 1127, 1058, 821, 754, 714, $691 ;{ }^{1} \mathrm{H}$ NMR $\left(500 \mathrm{MHz}, \mathrm{CDCl}_{3}\right) \delta 1.98\left(\mathrm{~s}, 3 \mathrm{H}, \mathrm{CH}_{3}\right), 3.28(\mathrm{~d}, J=$ $\left.4.0 \mathrm{~Hz}, 3 \mathrm{H}, \mathrm{NCH}_{3}\right), 5.36(\mathrm{~s}, 1 \mathrm{H}, \mathrm{CH}), 7.37$ (t, J= 7.5 Hz, 1H, ArH), 7.49-7.53 (m, 4H), 7.69 (d, $J=7.5 \mathrm{~Hz}, 2 \mathrm{H}, \mathrm{ArH}), 8.16$ (d, $J=$ $8.0 \mathrm{~Hz}, 2 \mathrm{H}, \mathrm{ArH}), 10.57$ (d, $J=2.0 \mathrm{~Hz}, 1 \mathrm{H}, \mathrm{NH}) ;{ }^{13} \mathrm{C}$ NMR $(125$ $\left.\mathrm{MHz} \mathrm{CDCl}_{3}\right) \delta 12.7,28.6,38.4$, 99.6, 109.4, 120.8, 123.5, 127.1, 129.1, 129.4, 137.2, 141.9, 146.0, 146.9, 149.3, 159.0; anal. calcd for $\mathrm{C}_{20} \mathrm{H}_{17} \mathrm{~N}_{5} \mathrm{O}_{5}$ : C, 58.97; H, 4.21; N, 17.19\%. Found: C, 58.90; $\mathrm{H}, 4.25$; N, $17.25 \%$.

4h: 4-(4-Methoxyphenyl)-N,3-dimethyl-5-nitro-1-phenyl-1,4dihydropyrano[2,3-c]pyrazol-6-amine. Isolated as white solid; yield: $87 \%$; mp: $210-212{ }^{\circ} \mathrm{C}$; IR $\left(\mathrm{KBr}, \mathrm{cm}^{-1}\right)$ : 3430, 3185, 1654, $1611,1515,1494,1448,1386,1355,1260,1128,756,{ }^{1} \mathrm{H}$ NMR $\left(500 \mathrm{MHz}_{\mathrm{CDCl}}\right) \delta 2.01\left(\mathrm{~s}, 3 \mathrm{H}, \mathrm{CH}_{3}\right), 3.23(\mathrm{~d}, J=4.9 \mathrm{~Hz}, 3 \mathrm{H}$, $\left.\mathrm{NCH}_{3}\right), 3.78\left(\mathrm{~s}, 3 \mathrm{H}, \mathrm{OCH}_{3}\right), 5.22(\mathrm{~s}, 1 \mathrm{H}, \mathrm{CH}), 6.82(\mathrm{~d}, J=8.0 \mathrm{~Hz}$, $2 \mathrm{H}, \operatorname{ArH}), 7.22$ (d, $J=8.0 \mathrm{~Hz}, 2 \mathrm{H}, \operatorname{ArH}), 7.34(\mathrm{t}, J=7.3 \mathrm{~Hz}, 1 \mathrm{H}$, $\operatorname{ArH}), 7.50$ (t, $J=7.5 \mathrm{~Hz}, 2 \mathrm{H}, \operatorname{ArH}), 7.70$ (d, $J=7.8 \mathrm{~Hz}, 2 \mathrm{H}, \operatorname{ArH})$, $10.56(\mathrm{~s}, 1 \mathrm{H}, \mathrm{NH}) ;{ }^{13} \mathrm{C} \mathrm{NMR}\left(125 \mathrm{MHz}, \mathrm{CDCl}_{3}\right) \delta 12.7,28.4,37.5$, 55.2, 101.3, 110.7, 113.6, 114.9, 120.7, 126.7, 129.3, 134.0, 137.5, 141.9, 146.4, 158.5, 159.1; anal. calcd for $\mathrm{C}_{21} \mathrm{H}_{20} \mathrm{~N}_{4} \mathrm{O}_{4}$ : C, 64.28; H, 5.14; N, 14.28\%. Found: C, 64.32; H, 5.17; N, 14.30\%.

4j: 4-(2-Chlorophenyl)- $N, 3-d i m e t h y l-5-n i t r o-1-p h e n y l-1,4-$ dihydropyrano[2,3-c]pyrazol-6-amine. Isolated as white solid; yield: $85 \%$; mp $244-245{ }^{\circ} \mathrm{C}$; IR $\left(\mathrm{KBr}, \mathrm{cm}^{-1}\right)$ : 3177, 3064, 2946, 2486, 2275, 1661, 1520, 1361, 1267, 1131, 908; ${ }^{1} \mathrm{H}$ NMR (400 MHz, DMSO- $\left.d_{6}\right) \delta 1.91\left(\mathrm{~s}, 3 \mathrm{H}, \mathrm{CH}_{3}\right), 3.20(\mathrm{~d}, J=4.8 \mathrm{~Hz}, 3 \mathrm{H}$, $\left.\mathrm{NCH}_{3}\right), 5.60(\mathrm{~s}, 1 \mathrm{H}, \mathrm{CH}), 7.17-7.22(\mathrm{~m}, 2 \mathrm{H}, \mathrm{ArH}), 7.32$ (d, $J=$ $7.6 \mathrm{~Hz}, 2 \mathrm{H}, \mathrm{ArH}), 7.49$ (t, $J=8.0 \mathrm{~Hz}, 2 \mathrm{H}, \mathrm{ArH}), 7.68-7.71(\mathrm{~m}, 3 \mathrm{H}$, ArH), 10.62 (d, $J=5.2 \mathrm{~Hz}, 1 \mathrm{H}, \mathrm{NH}) ;{ }^{13} \mathrm{C}$ NMR (100 MHz, DMSO$\left.d_{6}\right) \delta 12.4,28.5,38.8,108.1,120.2,120.5,126.4,126.9,128.0$, $129.1,132.5,137.0,141.8,145.1,158.7$; anal. calcd for $\mathrm{C}_{20} \mathrm{H}_{17} \mathrm{ClN}_{4} \mathrm{O}_{3}$ : C, 60.53; H, 4.32; N, 14.12\%. Found: C, 60.45; H, 4.37; N, $14.10 \%$.

4l: $N, 3-D i m e t h y l-5-n i t r o-4-(3-n i t r o p h e n y l)-1-p h e n y l-1,4-d i h y-$ dropyrano[2,3-c]pyrazol-6-amine. Isolated as white solid; yield: 86\%; mp 227-228 ${ }^{\circ} \mathrm{C}$; IR ( $\left.\mathrm{KBr}, \mathrm{cm}^{-1}\right): 3615,3178,3078,2924$, 1870, 1794, 1656, 1527, 1457, 1349, 1266, 1128, 1053, 927; ${ }^{1} \mathrm{H}$ NMR (400 MHz, DMSO- $\left.d_{6}\right) \delta 1.90\left(\mathrm{~s}, 3 \mathrm{H}, \mathrm{CH}_{3}\right), 3.19(\mathrm{~d}, J=$ $\left.5.2 \mathrm{~Hz}, 3 \mathrm{H}, \mathrm{NCH}_{3}\right), 5.46(\mathrm{~s}, 1 \mathrm{H}, \mathrm{CH}), 7.37$ (t, $\left.J=7.6 \mathrm{~Hz}, 1 \mathrm{H}, \mathrm{ArH}\right)$, 7.53-7.62 (m, 3H, ArH), 7.77 (d, $J=7.6 \mathrm{~Hz}, 2 \mathrm{H}, \mathrm{ArH}), 7.88(\mathrm{~d}, J=$ $8.0 \mathrm{~Hz}, 1 \mathrm{H}, \mathrm{ArH}$ ), 8.09 (dd, $J=8.4,1.2 \mathrm{~Hz}, 1 \mathrm{H}, \mathrm{ArH}), 8.27$ (s, $1 \mathrm{H}$, $\mathrm{ArH}), 10.67$ (d, $J=4.8 \mathrm{~Hz}, 1 \mathrm{H}, \mathrm{NH}) ;{ }^{13} \mathrm{C}$ NMR (100 MHz, DMSO$\left.d_{6}\right) \delta 12.5,28.7,37.6,99.4,108.1,120.4,121.7,122.4,126.7$, 129.4, 134.8, 137.0, 141.8, 145.3, 147.6, 158.6; anal. calcd for $\mathrm{C}_{20} \mathrm{H}_{17} \mathrm{~N}_{5} \mathrm{O}_{5}$ : C, 58.97; H, 4.21; N, 17.19\%. Found: C, 58.90; H, $4.25 ; \mathrm{N}, 17.20 \%$.

4n: $\quad N, 3-D i m e t h y l-5-n i t r o-1-p h e n y l-4-(3,4,5-$ trimethoxyphenyl)-1,4-dihydropyrano[2,3-c]pyrazol-6-amine. Isolated as white solid; yield: $89 \%$; mp $218-220{ }^{\circ} \mathrm{C}$; IR $\left(\mathrm{KBr}, \mathrm{cm}^{-1}\right): 3436$, 2947, 1655, 1620, 1595, 1495, 1355, 1234, 1127, 1054, 923; ${ }^{1} \mathrm{H}$ NMR (400 MHz, DMSO- $\left.d_{6}\right) \delta 2.08\left(\mathrm{~s}, 3 \mathrm{H}, \mathrm{CH}_{3}\right), 3.28(\mathrm{~d}, J=$ $\left.5.2 \mathrm{~Hz}, 3 \mathrm{H}, \mathrm{NCH}_{3}\right), 3.82\left(\mathrm{~s}, 3 \mathrm{H}, \mathrm{OCH}_{3}\right), 3.84\left(\mathrm{~s}, 6 \mathrm{H}, \mathrm{OCH}_{3}\right), 5.24$ $(\mathrm{s}, 1 \mathrm{H}, \mathrm{CH}), 6.51(\mathrm{~s}, 2 \mathrm{H}, \mathrm{ArH}), 7.34-7.38(\mathrm{~m}, 1 \mathrm{H}, \mathrm{ArH}), 7.52(\mathrm{t}, J=$ 16.0, 8.0 Hz, 2H, ArH), 7.70 (dd, $J=8.6 \mathrm{~Hz}, 1.0 \mathrm{~Hz}, 2 \mathrm{H}, \mathrm{ArH}$ ), $10.60(\mathrm{~d}, J=5.2 \mathrm{~Hz}, 1 \mathrm{H}, \mathrm{NH}) ;{ }^{13} \mathrm{C}$ NMR $\left(100 \mathrm{MHz}, \mathrm{DMSO}-d_{6}\right)$ $\delta$ 13.0, 28.6, 38.7, 56.2, 60.8, 100.8, 105.5, 110.2, 120.7, 126.9, $129.4,137.1,137.5,141.8,142.4,146.5,153.0,159.1$; anal. calcd 
for $\mathrm{C}_{23} \mathrm{H}_{24} \mathrm{~N}_{4} \mathrm{O}_{6}$ : C, 61.05; H, 5.35; N, 12.38\%. Found: C, 61.10; $\mathrm{H}, 5.37$, N, $12.40 \%$.

4o: $\quad N, 3-D i m e t h y l-5-n i t r o-1-p h e n y l-4-(p y r i d i n-3-y l)-1,4-d i h y-$ dropyrano[2,3-c]pyrazol-6-amine. Isolated as white solid; yield: 83\%; mp 220-221 ${ }^{\circ} \mathrm{C}$; IR ( $\left.\mathrm{KBr}, \mathrm{cm}^{-1}\right)$ : 3399, 3184, 3060, 2993, 2920, 1654, 1620, 1595, 1520, 1490, 1388, 1265, 925; ${ }^{1} \mathrm{H}$ NMR $\left(500 \mathrm{MHz}, \mathrm{CDCl}_{3}\right) \delta 1.98\left(\mathrm{~s}, 3 \mathrm{H}, \mathrm{CH}_{3}\right), 3.24(\mathrm{~d}, J=4.0 \mathrm{~Hz}, 3 \mathrm{H}$, $\left.\mathrm{NCH}_{3}\right), 5.26(\mathrm{~s}, 1 \mathrm{H}, \mathrm{CH}), 7.21(\mathrm{~d}, J=5.5 \mathrm{~Hz}, 1 \mathrm{H}, \mathrm{ArH}), 7.35(\mathrm{t}, J=$ $7.0 \mathrm{~Hz}, 1 \mathrm{H}, \mathrm{ArH}), 7.50$ (t, $J=7.2 \mathrm{~Hz}, 2 \mathrm{H}, \mathrm{ArH}), 7.62(\mathrm{~d}, J=6.5 \mathrm{~Hz}$, $1 \mathrm{H}, \mathrm{ArH}), 7.68$ (d, $J=7.5 \mathrm{~Hz}, 2 \mathrm{H}, \mathrm{ArH}), 8.45(\mathrm{~d}, J=3.5 \mathrm{~Hz}), 8.58$ (s, 1H, ArH), 10.55 (s, 1H, NH); ${ }^{13} \mathrm{C}$ NMR (125 MHz, $\mathrm{CDCl}_{3}$ ) $\delta$ 12.7, 28.5, 36.5, 99.9, 109.6, 114.9, 120.8, 123.2, 127.0, 129.4, 135.6, 137.3, 137.4, 142.0, 146.1, 148.3, 149.8, 159.0; anal. calcd for $\mathrm{C}_{19} \mathrm{H}_{17} \mathrm{~N}_{5} \mathrm{O}_{3}: \mathrm{C}, 62.80 ; \mathrm{H}, 4.72 ; \mathrm{N}, 19.27 \%$. Found: C, 62.85; $\mathrm{H}, 4.75$; N, $19.30 \%$.

6a: 2-(Methylamino)-3-nitro-4-phenylpyrano[3,2-c]chromen$\mathbf{5 ( 4 H )}$-one (6a). Isolated as yellow solid; yield: 91\%; mp: $278-$ $280^{\circ} \mathrm{C}$; IR ( $\left.\mathrm{KBr}, \mathrm{cm}^{-1}\right): 3438,3200,3061,2927,1727,1672,1629$, $1455,1358,1268,1154,1013,949 ;{ }^{1} \mathrm{H}$ NMR $\left(500 \mathrm{MHz}, \mathrm{DMSO}-d_{6}\right)$ $\delta 3.69\left(\mathrm{~s}, 3 \mathrm{H}, \mathrm{NCH}_{3}\right), 5.04(\mathrm{~s}, 1 \mathrm{H}, \mathrm{CH}), 6.820(\mathrm{~d}, J=7.0 \mathrm{~Hz}, 2 \mathrm{H}$, ArH), 7.24 (d, $J=7.0 \mathrm{~Hz}, 2 \mathrm{H}, \operatorname{ArH}), 7.48-7.52$ (m, 3H, ArH), 7.73 $(\mathrm{t}, J=7.0 \mathrm{~Hz}, 1 \mathrm{H}, \mathrm{ArH}), 8.01(\mathrm{~d}, J=7.0 \mathrm{~Hz}, 1 \mathrm{H}, \mathrm{ArH}), 10.36(\mathrm{~s}$, $1 \mathrm{H}, \mathrm{NH}) ;{ }^{13} \mathrm{C}$ NMR $\left(125 \mathrm{MHz}, \mathrm{DMSO}-d_{6}\right) \delta 29.1,36.7,55.5,107.3$, $108.4,113.1,113.9$, 114.9, 117.0, 123.2, 125.4, 129.9, 133.5, 152.1, 152.4 157.2, 158.7, 159.6; anal. calcd for $\mathrm{C}_{19} \mathrm{H}_{14} \mathrm{~N}_{2} \mathrm{O}_{5}$ : C, 65.14; H, 4.03; N, 8.00\%. Found: C, 65.85; H, 4.07; N, 8.03\%. EIHRMS: anal. calcd for $\left[\mathrm{C}_{19} \mathrm{H}_{14} \mathrm{~N}_{2} \mathrm{O}_{5}+\mathrm{H}^{+}\right]$: cacld: 351.0936, found: 351.0979 .

6b: 4-(4-Chlorophenyl)-2-(methylamino)-3-nitropyrano[3,2-c] chromen-5(4H)-one. Isolated as white solid; yield: 92\%; mp: 256-258 ${ }^{\circ} \mathrm{C}$; IR (KBr, cm ${ }^{-1}$ ) 3188, 2978, 2834, 1731, 1242, 1125, 1606, 1426; ${ }^{1} \mathrm{H}$ NMR (400 MHz, DMSO- $\left.d_{6}\right) \delta 3.32(\mathrm{~d}, J=4.0 \mathrm{~Hz}$, $\left.3 \mathrm{H}, \mathrm{NCH}_{3}\right), 5.15(\mathrm{~s}, 1 \mathrm{H}, \mathrm{CH}), 7.45(\mathrm{~d}, J=5.6 \mathrm{~Hz}, 1 \mathrm{H}, \mathrm{ArH}), 7.50(\mathrm{t}$, $J=5.2 \mathrm{~Hz}, 1 \mathrm{H}, \mathrm{ArH}), 7.65(\mathrm{~d}, J=6.0,2 \mathrm{H}, \mathrm{ArH}), 7.62-7.77(\mathrm{~m}, 1 \mathrm{H}$, $\operatorname{ArH}), 8.0$ (d, $J=5.2 \mathrm{~Hz}, \operatorname{ArH}), 8.10$ (d, $J=6.0 \mathrm{~Hz}, 2 \mathrm{H}, \operatorname{ArH}), 10.5$ $(\mathrm{d}, J=3.2 \mathrm{~Hz}, 1 \mathrm{H}, \mathrm{NH}) ;{ }^{13} \mathrm{C}$ NMR $\left(100 \mathrm{MHz}, \mathrm{DMSO}-d_{6}\right) \delta 29.1$, 38.1, 105.6, 107.4, 112.8, 117.0, 123.4, 123.5, 125.6, 127.1, 130.5, 133.9, 146.9, 149.1, 152.5, 152.7, 157.0, 159.7. Anal. calcd for $\mathrm{C}_{19} \mathrm{H}_{13} \mathrm{ClN}_{2} \mathrm{O}_{5}$ : C, 59.31; H, 3.41; N, 7.28\%. Found: C, 59.35; H, 3.43 ; N, $7.31 \%$.

6d: 4-(4-Fluorophenyl)-2-(methylamino)-3-nitropyrano[3,2-c] chromen-5(4H)-one. Isolated as yellow solid; yield: $86 \%$; mp: 262-264 ${ }^{\circ} \mathrm{C}$; IR (KBr, cm ${ }^{-1}$ ): 3421, 3066, 2942, 1722, 1672, 1505, 1384, 1271, 1158, 1069, 908; ${ }^{1} \mathrm{H}$ NMR (400 MHz, DMSO- $d_{6}$ ) $\delta 3.33\left(\mathrm{~d}, J=5.04 \mathrm{~Hz}, 3 \mathrm{H}, \mathrm{NCH}_{3}\right), 5.07(\mathrm{~s}, 1 \mathrm{H}, \mathrm{CH}), 7.08(\mathrm{t}, J=$ $8.8 \mathrm{~Hz}, 2 \mathrm{H}, \mathrm{ArH}), 7.38$ (dd, $J=5.3 \mathrm{~Hz}, 2 \mathrm{H}, \mathrm{ArH}), 7.51$ (q, $J=$ $7.6 \mathrm{~Hz}, 2 \mathrm{H}, \mathrm{ArH}), 7.74(\mathrm{~m}, 1 \mathrm{H}, \mathrm{ArH}), 8.0(\mathrm{dd}, J=8.0,1.6 \mathrm{~Hz}, 1 \mathrm{H}$, ArH), 10.4 (d, $J=4.72,1 \mathrm{H}, \mathrm{NH}) ;{ }^{13} \mathrm{C}$ NMR (100 MHz, DMSO- $\left.d_{6}\right)$ $\delta 28.6,36.8,106.3,107.6,112.5,114.6,114.8,116.5,122.8,125.0$, $130.4,130.5$, 133.1, 137.3, 151.8, 151.9, 156.6, 159.1, 159.9, 162.3; anal. calcd for $\mathrm{C}_{19} \mathrm{H}_{13} \mathrm{FN}_{2} \mathrm{O}_{5}$ : C, 61.96; H, 3.56; N, 7.61\%. Found: C, 61.91; H, 3.51; N, 7.60\%.

6h: 4-(4-Ethylphenyl)-2-(methylamino)-3-nitropyrano[3,2-c] chromen-5(4H)-one. Isolated as yellow solid; yield: $83 \%$; mp: 256-258 ${ }^{\circ} \mathrm{C}$; IR (KBr, $\mathrm{cm}^{-1}$ ): 3213, 3022, 2961, 2932, 2871, 1734, $1674,1630,1460,1569,1360,1268,1106,948,839 ;{ }^{1} \mathrm{H}$ NMR $(400$ MHz, DMSO- $\left.d_{6}\right) \delta 1.12(\mathrm{t}, J=7.7 \mathrm{~Hz}, 3 \mathrm{H}), 2.53(\mathrm{t}, J=7.6 \mathrm{~Hz}, 2 \mathrm{H})$, $3.32\left(\mathrm{~d}, J=5 \mathrm{~Hz}, 3 \mathrm{H}, \mathrm{NCH}_{3}\right), 5.0(\mathrm{~s}, 1 \mathrm{H}, \mathrm{CH}), 7.0(\mathrm{~d}, J=8.2 \mathrm{~Hz}$, $2 \mathrm{H}, \operatorname{ArH}), 7.23$ (d, J=8.2 Hz, 2H, ArH), 7.49 (m, 2H, ArH), 7.73 (m, 1H, ArH), 8.0 (dd, $J=7.9,1.5 \mathrm{~Hz}, 1 \mathrm{H}, \mathrm{ArH}), 10.39$ (d, $J=$ $4.8 \mathrm{~Hz}, 1 \mathrm{H}) ;{ }^{13} \mathrm{C}\left(100 \mathrm{MHz}, \mathrm{CDCl}_{3}\right) \delta 15.5,27.7,28.6,103.6$, $106.7,107.8,112.5,115.5,116.5,122.7,125.0$, 127.1, 127.4, 128.3, 133.1, 138.5, 142.5, 151.7, 151.9, 152.3, 156.7, 159.2. Anal. calcd for $\mathrm{C}_{21} \mathrm{H}_{18} \mathrm{~N}_{2} \mathrm{O}_{5}: \mathrm{C}, 66.66 ; \mathrm{H}, 4.79 ; \mathrm{N}, 7.40 \%$. Found: $\mathrm{C}$, 66.18; H, 4.77; N, 7.35\%.

6j: 2-(Methylamino)-3-nitro-4-(3-nitrophenyl)pyrano[3,2-c] chromen-5(4H)-one. Isolated as light yellow solid; yield: $81 \%$; $\mathrm{mp} 258-260{ }^{\circ} \mathrm{C}$; IR $\left(\mathrm{KBr}, \mathrm{cm}^{-1}\right): 2927,1726,1674,1631,1460$, $1272,1163,1105,1072 ;{ }^{1} \mathrm{H}$ NMR $\left(400 \mathrm{MHz}, \mathrm{DMSO}-d_{6}\right) \delta 3.32(\mathrm{~d}, J$ $\left.=5.0,3 \mathrm{H}, \mathrm{NCH}_{3}\right), 6.07(\mathrm{~s}, 1 \mathrm{H}, \mathrm{CH}), 7.47-7.59(\mathrm{~m}, 5 \mathrm{H}, \mathrm{ArH}), 7.74$ (m, 1H, ArH) 7.90-7.93, (dd, $J=8.2,2.3, \mathrm{~Hz}, 1 \mathrm{H}, \mathrm{ArH}), 8.03$ (dd, $J=8.0,1.6 \mathrm{~Hz}, 1 \mathrm{H}, \mathrm{ArH}), 10.51(\mathrm{~d}, J=5.2 \mathrm{~Hz}, 1 \mathrm{H}, \mathrm{NH}) ;{ }^{13} \mathrm{C} \mathrm{NMR}$ (100 MHz, DMSO- $d_{6}$ ) $\delta$ 28.7, 105.2, 107.0, 116.6, 118.2, 122.1, $123.0,123.3$, 125.0, 129.4, 133.4, 135.4, 143.3, 147.3, 152.2, 156.6, 159.4; anal. calcd for $\mathrm{C}_{19} \mathrm{H}_{13} \mathrm{~N}_{3} \mathrm{O}_{7}$ : C, 57.73; H, 3.31; N, $10.63 \%$. Found: C, 57.77; H, 3.28; N, 10.60\%.

61: 4-(3-Chlorophenyl)-2-(methylamino)-3-nitropyrano[3,2-c] chromen-5(4H)-one. Isolated as yellow solid; yield: $85 \%$; $\mathrm{mp}$ : 270-272 ${ }^{\circ} \mathrm{C}$; IR (KBr, cm ${ }^{-1}$ ): 3206, 2926, 2855, 1729, 1610, 1435, $1015 ;{ }^{1} \mathrm{H}$ NMR (400 MHz, DMSO- $d_{6}$ ), $\delta 3.33$ (d, $J=4.8 \mathrm{~Hz}, 3 \mathrm{H}$, $\left.\mathrm{NCH}_{3}\right), 5.06(\mathrm{~s}, 1 \mathrm{H}, \mathrm{CH}), 7.25-7.34(\mathrm{~m}, 3 \mathrm{H}, \mathrm{ArH}), 7.41(\mathrm{~s}, 1 \mathrm{H})$, 7.47-7.53 (m, 2H, ArH), 7.72-7.76 (m, 1H), $8.0(\mathrm{dd}, J=7.6$, $1.2 \mathrm{~Hz}, 1 \mathrm{H}), 10.44$ (d, $J=4.8 \mathrm{~Hz}, 1 \mathrm{H}) ;{ }^{13} \mathrm{C} \mathrm{NMR}(100 \mathrm{MHz}$, DMSO- $\left.d_{6}\right) \delta 28.7,37.4,105.7,107.2,112.5,116.5,122.9,124.9$, $127.0,127.3,128.4,129.8,132.5$, 133.2, 143.5, 152.0, 156.5, 159.1; anal. calcd for $\mathrm{C}_{19} \mathrm{H}_{13} \mathrm{ClN}_{2} \mathrm{O}_{5}$ : C, 59.31; H, 3.41; N, 7.28\%. Found: C, 59.27; H, 3.39; N, 7.35\%.

\section{Conflicts of interest}

There are no conflicts to declare.

\section{Acknowledgements}

S. Shareef and Saigal are grateful to CSIR, New Delhi for their research fellowship. The authors would like to thanks SAIF-PU, Panjab University, Chandigarh and SAIF, IIT Roorkee, IIT Ropar for providing the spectral data such as ${ }^{1} \mathrm{H}$ and ${ }^{13} \mathrm{C}$ NMR, HRMS, elemental analysis and SC Sahoo thanks DST-FIST for the single crystal XRD facility at Panjab University Chandigarh. We are also obliged to the Chairman, Department of Chemistry, AMU, Aligarh for providing general facility to execute this task.

\section{Notes and references}

1 (a) R. B. Carvalho and S. V. Joshi, Green Chem., 2019, 21, 1921; (b) Y. Gu and F. Jerôme, Green Chem., 2010, 12, 1127; (c) P. J. Dunn, Chem. Soc. Rev., 2012, 41, 1452.

2 (a) P. T. Anastas and J. C. Warner, Green Chemistry: Theory and Practice, Oxford University Press Inc., 1st edn, New York, 1998; (b) M. M. Kirchhoff, Environ. Sci. Technol., 2003, 37, 5349; (c) I. T. Horvath and P. T. Anastas, Chem. Rev., 2007, 107, 2169. 
3 (a) M. S. Singh and S. Chowdhury, RSC Adv., 2012, 2, 4547; (b) A. Kumar and S. A. Sharma, Green Chem., 2011, 13, 2017.

4 (a) M. M. Khan, S. Khan, Saigal and S. Iqbal, RSC Adv., 2016, 6, 42045; (b) A. T. Khan, D. K. Das and M. M. Khan, Tetrahedron Lett., 2011, 52, 4539; (c) R. C. Cioc, E. Ruijter and R. V. A. Orru, Green Chem., 2014, 16, 2958; (d) A. T. Khan, M. M. Khan, D. K. Das and M. Lal, J. Heterocycl. Chem., 2012, 49, 1362.

5 (a) H. G. O. Alvim, J. R. Correa, J. A. F. Assumpcao, W. A. da Silva, M. O. Rodrigues, J. L. de Macedo, M. Fioramonte, F. C. Gozzo, C. C. Gatt and B. A. D. Neto, J. Org. Chem., 2018, 83, 4044; (b) H. G. O. Alvim, D. L. J. Pinheiro, V. H. Carvalho-Silva, M. Fioramonte, F. C. Gozzo, W. A. da Silva, G. W. Amarante and B. A. D. Neto, J. Org. Chem., 2018, 83, 12143; (c) L. Zeng, B. Huang, Y. Shen and S. Cui, Org. Lett., 2018, 20, 3460.

6 (a) P. N. Kalaria, S. C. Karad and D. K. Raval, Eur. J. Med. Chem., 2018, 158, 917; (b) A. K. Mourad, F. K. Mohamed and A. Y. Soliman, Heterocycles, 2018, 96, 50; (c) Saigal, S. Shareef, H. Rahman and M. M. Khan, Curr. Org. Chem., 2019, 23, 1045.

7 (a) S. C. Huang, P. L. Wu and T. S. Wu, Phytochemistry, 1997, 44, 179; (b) C. R. Su, S. F. Yeh and C. M. Liu, Bioorg. Med. Chem., 2009, 17, 6137; (c) D. K. Yadav and M. A. Quraishi, Ind. Eng. Chem. Res., 2012, 51, 8194.

8 (a) M. Klein, P. Diner, D. Dorin-Semblat, C. Doerig and M. Grotli, Org. Biomol. Chem., 2009, 7, 3421; (b) S. M. Deshmukh and D. P. Hiwarale, Der Pharma Chem., 2017, 9, 109.

9 (a) B. S. Dawane, O. S. Yemul, S. S. Chobe, G. G. Mandawad, R. D. Kamble, A. V. Shinde, V. S. Kale, A. O. Hurne, M. A. Pawde, M. P. Kale, N. P. Desai, R. R. Salgare, M. B. Patil, S. N. Mundhe and S. R. Chavan, Der Pharma Chem., 2011, 3, 300; (b) S. Ahadi, Z. Yasaei and A. Bazgir, J. Heterocycl. Chem., 2010, 47, 1090.

10 (a) M. T. Flavin, J. D. Rizzo, A. Khilevich, A. Kucherenko, A. K. Sheinkman, V. Vilaychack, L. Lin, W. Chen, E. M. Greenwood, T. Pengsuparp, J. M. Pezzuto, S. H. Hughes, T. M. Flavin, M. Cibulski, W. A. Boulanger, R. L. Shone and Z. Q. Xu, J. Med. Chem., 1996, 39, 1303; (b) V. F. De Andrade-Neto, M. O. Goulart, J. F. Da Silva Filho, M. J. Da Silva, M. D. C. Pinto, A. V. Pinto, M. G. Zalis, L. H. Carvalho and A. U. Krettli, Bioorg. Med. Chem. Lett., 2004, 14, 1145.

11 A. Kumar, R. A. Maurya, S. A. Sharma, P. Ahmad, A. B. Singh, G. Bhatia and A. K. Srivastava, Bioorg. Med. Chem. Lett., 2009, 19, 6447.
12 S. J. Vaghasiya, D. K. Dodiya, A. R. Trivedi and V. H. Shah, ARKIVOC, 2008, (xii), 1.

13 Y. L. Zhang, B. Z. Chen, K. Q. Zheng, M. L. Xu, X. H. Lei and X. B. Yaoxue, Chem. Abstr., 1982, 96, 135383.

14 E. Melliou, P. Magiatis, S. Mitaku, A.-L. Skaltsounis, E. Chinou and I. Chinou, J. Nat. Prod., 2005, 68, 78.

15 (a) M. A. Al-Haiza, M. S. Mostafa and M. Y. El-Kady, Molecules, 2003, 8, 275; (b) P. C. B. Page, L. F. Appleby, D. Day, Y. Chan, B. R. Buckley, S. M. Allim and M. J. Mckenzie, Org. Lett., 2009, 11, 1991.

16 A. Lacy and R. O' Kennedy, Curr. Pharm. Des., 2004, 10, 3797. 17 (a) P. N. Kalaria, S. P. Satasia and D. K. Raval, New J. Chem., 2014, 38, 1512; (b) D. C. Mungra, M. P. Patel, D. P. Rajani and R. G. Patel, Eur. J. Med. Chem., 2011, 46, 4192.

18 S. A. El-Assiery, G. H. Sayed and A. Fouda, Acta Pharm., 2004, 54, 143.

19 (a) Saigal, S. Khan, H. Rahman, Shafiullah and M. M. Khan, RSC Adv. , 2019, 9, 14477; (b) F. Rahimi, H. Hosseini, M. Bayat and Y. T. Jeong, Tetrahedron, 2018, 59, 818; (c) A. M. Jadhav, Y. I. Kim, K. T. Lim and Y. T. Jeong, Tetrahedron, 2018, 59, 554; (d) A. Chaudhary, J. M. Khurana, G. Khanna and M. Saroha, ChemistrySelect, 2018, 3, 6334; (e) B. Balachandra and S. Shanmugam, ChemistrySelect, 2018, 3, 2037.

20 D. N. Survase, H. V. Chavan, S. B. Dongare, S. D. Ganapure and V. B. Helavi, Iran. Chem. Commun., 2017, 5, 105.

21 K. Jayabal and T. P. Paramasivan, Tetrahedron Lett., 2014, 55, 2010.

22 A. M. Jadhav, S. K. Krishnammagari, J. T. Kim and Y. T. Jeong, Tetrahedron, 2017, 73, 5163.

23 V. V. Shinde, D. Jeong, E. Cho and S. Jung, Tetrahedron, 2018, 74, 194.

24 J. Kamalraja, D. Muralidharan and P. T. Perumal, Synlett, 2012, 23, 2894.

25 (a) M. M. Khan, B. Saigal, S. Shareef, S. Khan and S. C. Sahoo, Synth. Commun., 2018, 48, 2683; (b) M. M. Khan, Saigal, S. Khan, S. Shareef and S. C. Sahoo, RSC Adv., 2018, 8, 41892; (c) M. M. Khan, Saigal and S. Khan, J. Heterocycl. Chem., 2019, 56, 1020.

26 (a) M. M. Khan, Saigal, S. Khan, S. Shareef and M. Danish, ChemistrySelect, 2018, 3, 6830; (b) M. M. Khan, Saigal, S. Khan, S. Shareef and S. Hussain, ChemistrySelect, 2018, 3, 2261; (c) M. M. Khan, S. Khan, Saigal and S. C. Sahoo, ChemistrySelect, 2018, 3, 1371; (d) M. M. Khan, S. Khan, S. Iqbal, Saigal and R. Yousuf, New J. Chem., 2016, 40, 7504. 